\title{
Positive Interval Observer-based State Feedback Controller for Uncertain General Anaesthesia System
}

\author{
Jing Jing Chang1, S. Syafiie ${ }^{2}$ \\ ${ }^{1}$ Faculty of Information and Communication Technology, Universiti Tunku Abdul Rahman, Malaysia \\ ${ }^{2}$ Faculty of Engineering, Syiah Kuala University, Indonesia
}

\begin{abstract}
Article Info
Article history:

Received Jan 11, 2018

Revised Mar 20, 2018

Accepted Apr 5, 2018

Keywords:

Depth of anaesthesia Inter-individual variability

ABSTRACT

The drug delivery process of general anaesthesia in the human body is most commonly described by the Pharmacokinetic/Pharmacodynamic (PK/PD) model. Since the PK model is a positive linear system, the design of the controller can be treated as a positive stabilization problem. In this paper, a state feedback controller with positive interval observer was designed using a linear programming approach by taking into account the inter-individual variability among patient in the PK model. The designed controller was assessed by simulation on a pool of patients. The result shows that the design of a fix controller for the whole population is difficult due to the conflict between performance and robustness.
\end{abstract}

Output feedback control

Positive linear system

Positive observer

Copyright (C) 2018 Institute of Advanced Engineering and Science. All rights reserved.

\section{Corresponding Author:}

Jing Jing Chang,

Department of Computer and Communication Technology,

Faculty of Information and Communication Technology,

Universiti Tunku Abdul Rahman,

Jalan Universiti Bandar Barat, 31900 Kampar, Perak, Malaysia.

Email: changjj@utar.edu.my

\section{INTRODUCTION}

The drug delivery process of general anaesthesia is most commonly described by the Pharmacokinetic/Pharmacodynamic (PK/PD) model. The PK model is a linear time invariant (LTI) model that relates the drug dosage to drug concentration in blood plasma, while PD model is a static nonlinear model that relates the drug concentration in the blood plasma to the resulting drug effect [1].

One important property of the PK model is that it is a positive system; the state variables (drug concentration in each compartment) can never be less than zero. Imposing positiveness in the design of closed-loop system allows one to simplify the stability analysis [2] and reduces the problem to a real bounded uncertainty gain problem [3]. Therefore, the control of general anaesthesia can be treated as a stabilization problem of a positive system.

Closed-loop stabilization of positive linear system has been considered in several previous works. For example, Kaczorek [4] proposed a sufficient condition for state feedback controller based on Gersgorin's theorem and quadratic programming. Later, Leenheer and Aeyels [5] solve the stabilisation of a state feedback using algebraic approach. Boyd et al [6] and Gao et al [7] proposed a necessary and sufficient condition to stabilised the closed-loop positive linear system formulated as Linear Matrix Inequality (LMI). Rami et al [8-9] also proposed a feedback stabilisation based on linear programming (LP) method.

The LP approach offers several advantages over the other methods: it provides both the necessary and sufficient condition to guarantee asymptotic stability of the system, it has a lower computational complexity compared to quadratic programming or LMI, and it can be extended easily to uncertain plants. 
Moreover, the LP inequalities can be used to design a positive interval observer [10]. In anaesthesia control, the drug concentration in each compartment was not possible to be measured in real time. Hence, an observer is needed to estimate the states. However, the classical Luenberger observer does not guarantee the positiveness of the estimated states. This can be achieved by using the LP approach that guarantees the estimated states to remain in the nonnegative orthant.

Conventionally, state feedback controllers have been designed using the LMI [11] or pole placement [12-13] methods. However, for problem with only partial information on the state is available, static output stabilization problem has not been formulated and solved exactly as an LMI problem or pole placement approach [8]. Furthermore, it can be difficult to use pole placement method for positive system since it is not even know which poles are desireable to ensure positivity of the closed-loop system. Hence, the LP approach has the advantage of easy handling of static output stabilization problem for positive systems.

In this paper, uncertainty was assumed to be occurred in PK model. LP approach was used to design the state-feedback controller and the positive interval observer, by taking into account the possible variability of parameters in the PK model.

\section{RESEARCH METHOD}

Several important notations are stated here. $\mathbf{R}^{\mathrm{n}}+$ denotes the nonnegative orthant of the $\mathrm{n}$ dimensional real space $\mathbf{R}^{\mathrm{n}}$. $M^{\top}$ denotes the transpose of the matrix $M$. Each elements of matrix $M$ is represented by $m_{i j}$ where $i$ is the number of row and $j$ is the number of column. $M_{i j} \geq 0$ means that all the elements are nonnegative. $\bar{M}$ and $\underline{M}$ represents the upper bound and lower bound of the uncertain matrix $\mathrm{M}$.

$$
\begin{aligned}
& x=A x+B u \\
& y=C x
\end{aligned}
$$

\section{a. Plant model}

The PK model can be described as a four-compartment model and expressed in the following state space Equation:

$$
\text { where } x=\left[\begin{array}{c}
C_{1} \\
C_{2} \\
C_{3} \\
C_{e}
\end{array}\right], A=\left[\begin{array}{cccc}
-k_{10}-k_{12}-k_{13} & k_{21} \frac{V_{2}}{V_{1}} & k_{31} \frac{V_{3}}{V_{1}} & 0 \\
k_{12} \frac{V_{1}}{V_{2}} & -k_{21} & 0 & 0 \\
k_{13} \frac{V_{1}}{V_{3}} & 0 & -k_{31} & 0 \\
k_{e 0} & 0 & 0 & -k_{e 0}
\end{array}\right], B=\left[\begin{array}{c}
\rho \\
\alpha V_{1} \\
0 \\
0 \\
0
\end{array}\right] \text { and } C=\left[\begin{array}{llll}
0 & 0 & 0 & 1
\end{array}\right] \text {, }
$$

$C_{1}, C_{2}, C_{3}$ and $C_{e}$ denote the drug concentration in compartment $1,2,3$ and effect compartment, respectively. $V_{i}$ is the volume for compartment $i$ while $k_{i j}$ is the drug transfer rate from compartment $i$ to $j$. $u$ is the propofol infusion rate $(\mathrm{ml} / \mathrm{h}), \rho$ is the drug concentration (for propofol, $\rho=10 \mathrm{mg} / \mathrm{ml}$ ) and $\alpha$ is a normalisation constant $(\alpha=60 \mathrm{~min} / \mathrm{h})$.

For Schnider PK model, the nominal value of compartment volumes and drug transfer rates can be found in [14-15]. However, due to inter-patient variability, it is expected that the true values will be different from the nominal values. Luckily, in most of the biological modellings, inter-variability remains bounded with a priori known bounds [16]. Consider a 50 year old male patient weighting $70 \mathrm{~kg}$ and $170 \mathrm{~cm}$ tall. A possible lower and upper bound of matrix A were given as the follow:

$$
\underline{A}=\left[\begin{array}{cccc}
-0.9689 & 0.3190 & 0.1958 & 0 \\
0.0679 & -0.0679 & 0 & 0 \\
0.0035 & 0 & -0.0035 & 0 \\
0.2420 & 0 & 0 & -0.4820
\end{array}\right], \bar{A}=\left[\begin{array}{cccc}
-0.7979 & 0.3190 & 0.1958 & 0 \\
0.0679 & -0.0679 & 0 & 0 \\
0.0035 & 0 & -0.0035 & 0 \\
0.2420 & 0 & 0 & -0.2420
\end{array}\right] \text {, }
$$

$\mathrm{B}$ and $\mathrm{C}$ were assumed certain and were equal to $B=\left[\begin{array}{llll}0.039 & 0 & 0 & 0\end{array}\right]^{T}$ and $C=\left[\begin{array}{llll}0 & 0 & 0 & 1\end{array}\right]$. PD model can be expressed by the following static nonlinear Hill equation: 
$B I S=B I S_{0}+\left(B I S_{\max }-B I S_{0}\right) \frac{C_{e}^{\gamma}}{C_{e 50}^{\gamma}+C_{e}^{\gamma}}$

where BIS is measurable parameter of anaesthetic level, $\mathrm{BIS}_{0}$ is the base line effect in the absence of drug, $\mathrm{BIS}_{\max }$ is the maximum effect of the drug, $C_{e 50}$ is the concentration that produces $50 \%$ of the maximum drug effect and $\gamma$ determine the steepness of the Hill equation. In this paper, it is assumed that $\mathrm{BIS}_{0}=100$, $\mathrm{BIS}_{\max }=0, C_{e 50}=2.23$ and $\gamma=1.72$. Due to the static nature of PD model, the nonlinear effect can be cancelled out by its inverse, $\mathrm{PD}^{-1}$. Hence, the plant can be controlled using linear control strategy.

\section{b. State feedback stabilisation}

First, it is assumed that the states are available for feedback. The objective is to determine the gain matrix $K$ in the control law $u=K x$ that stabilizes the system $\&=A x+B u$ while guaranteeing the system positiveness. According to [8], the $K$ can be determined such that $A+B K$ is both a Metzler and a Hurwitz matrix. These can be achieved by the following LP inequalities:

$$
\begin{aligned}
\bar{A} d+B \sum_{i=1}^{n} v_{i} & <0 \\
\underline{A} d+B \sum_{i=1}^{n} v_{i} & <0 \\
-d & <0
\end{aligned}
$$

And

$$
\begin{aligned}
-v_{i} & \leq 0 \\
-\sum_{i=1}^{n} v_{i} \leq 0 & \\
-\bar{a}_{i j} d_{j}-b_{i} v_{j} \leq 0 & \text { for } i \neq j \\
-\underline{a}_{i j} d_{j}-b_{i} v_{j} \leq 0 & \text { for } i \neq j
\end{aligned}
$$

where $K$ was computed as

$$
K=\left[d_{1}^{-1} v_{1} \mathrm{~K} d_{n}^{-1} v_{n}\right]
$$

\section{c. Positive interval observer}

Consider the following observer system

$$
\begin{aligned}
& \&=A x \\
& y=C x
\end{aligned}
$$

where the trajectory of $x \in \mathbf{R}_{+}^{n}$ is assumed to be unknown but nonnegative. Based on the classical Luenberger observer [17], the following linear observer was used to approximate the states $\hat{x}$

$$
\mathscr{L}=(A-L C) \hat{x}+L y
$$

where $\mathrm{L}$ is the observer gain. The aim here is to determine the observer gain $L$ such that the approximated states $\hat{x}$ are nonnegative and the error $\hat{x}-x$ converges asymptotically to zero.

According to [10], a positive interval observer can be designed using the following inequalities: 


$$
\begin{aligned}
\bar{A}^{T} d-C^{T} \sum_{i=1}^{n} z_{i} & <0 \\
-d & <0 \\
-c_{i}^{T} z_{j} & \leq 0 \quad \text { for } i, j=1, \mathrm{~K} n \\
-\underline{A}^{T} \operatorname{diag}(d)+C^{T} Z-\beta I & \leq 0 \\
-z_{i} & \leq 0
\end{aligned}
$$

with $L$ calculated as

$$
L=\left[d_{1}^{-1} z_{1} \mathrm{~K} d_{n}^{-1} z_{n}\right]
$$

\section{d. Integral action}

To include reference tracking and improve its disturbance rejection abilities, the control law was expanded to include integral action

$$
\begin{aligned}
& u=-K x-k_{i} z \\
& \&=y-r
\end{aligned}
$$

where $r$ is the reference point and $k_{i}$ as the integral term. The augmented system of the closed-loop controller was expressed as follows:

$$
\left[\begin{array}{l}
\& \\
\& \\
\&
\end{array}\right]=\left[\begin{array}{ccc}
A & 0 & 0 \\
L C & A-L C & 0 \\
C & 0 & 0
\end{array}\right]\left[\begin{array}{l}
x \\
\hat{x} \\
z
\end{array}\right]+\left[\begin{array}{l}
B \\
B \\
0
\end{array}\right] u+\left[\begin{array}{c}
0 \\
0 \\
-I
\end{array}\right] r
$$

Figure 1 shows the overall block diagram of the closed-loop system. The plant model has the propofol infusion rate, $\mathrm{u}$ as the input and Bispectral Index (BIS) as the output. BIS is the was assumed unknown. BIS is a widely used index that measures the depth of anaesthesia. The nonlinear effect introduced Since the patients were assumed unknown, the nominal matrix A was used in the observer block during simulation.

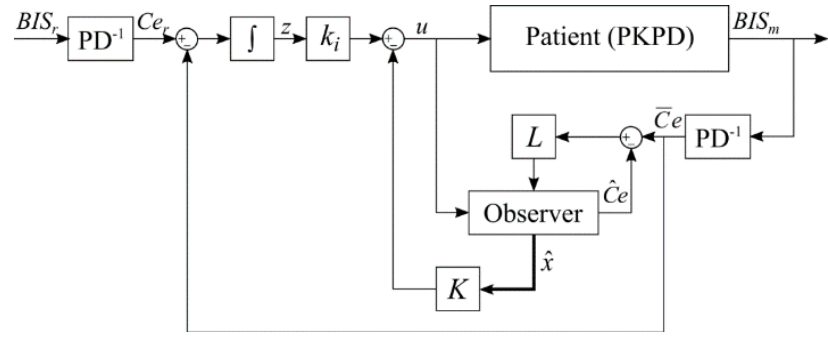

Figure 1. Block diagram of the observer-based state feedback control with integral action

\section{RESULTS AND ANALYSIS}

Based on the method described in Section 2, the controller gains were designed and computed to be $K=\left[\begin{array}{llll}0.3593 & 0.3660 & 0.3759 & 0.5103\end{array}\right], L=\left[\begin{array}{llll}0 & 0 & 0 & 0.0464\end{array}\right]$, and $k_{i}=2$. The designed controller was tested on 9 simulated patients with varying $k_{10}$ and $k_{e 0}$ values, as tabulated in Table 1 . These two values were chosen due to their higher impact on the variability of BIS effect.

The objective of the controller is to bring the BIS value down to 50, which is the recommended index during surgical procedures. The controller was assessed by its induction phase duration (ID), percentage of overshoot (OS), and integrated absolute error (IAE) [18]. The ID was defined as the time elapsed from the beginning of propofol administration until the BIS falls to below 60 for 30s [19]. A short ID is preferable because it reduces the time spent in the operating room. OS measures the degree of overshoot 
and should be as low as possible to prevent drug overdose. IAE is the integration of absolute difference between the BIS set-point and the real BIS.

Table 1. Test Population

\begin{tabular}{ccl}
\hline Patient no. & $k_{10}\left(\mathrm{~min}^{-1}\right)$ & $k_{e 0}\left(\mathrm{~min}^{-1}\right)$ \\
\hline 1 & 0.283 & 0.242 \\
2 & 0.283 & 0.362 \\
3 & 0.283 & 0.482 \\
4 & 0.369 & 0.242 \\
5 (Nominal) & 0.369 & 0.362 \\
6 & 0.369 & 0.482 \\
7 & 0.454 & 0.242 \\
8 & 0.454 & 0.362 \\
9 & 0.454 & 0.482 \\
\hline
\end{tabular}

Table 2 and Figure 2 shows the simulation result of the controller for all patients. Result shows that all BIS converges to the targeted value. The mean value of OS is $13.01 \%$, with no BIS lower than 40 . However, a long ID, with the mean of $14.78 \mathrm{~min}$, was observed. This duration was longer to $3 \mathrm{~min}$, which is considered too long for clinical practice and other research [20].

Table 2. Performance of the controller without initial bolus

\begin{tabular}{cccc}
\hline Patient no. & ID $(\mathrm{min})$ & OS $(\%)$ & IAE \\
\hline 1 & 14.50 & 19.42 & 13629 \\
2 & 13.50 & 14.70 & 11321 \\
3 & 13.08 & 12.34 & 10564 \\
4 & 15.50 & 16.33 & 12225 \\
5 & 14.58 & 12.08 & 10458 \\
6 & 14.25 & 10.12 & 9726 \\
7 & 16.50 & 13.77 & 11211 \\
8 & 15.67 & 9.98 & 9704 \\
9 & 15.42 & 8.37 & 9021 \\
\hline Mean & 14.78 & 13.01 & 10873 \\
\hline
\end{tabular}

(a)

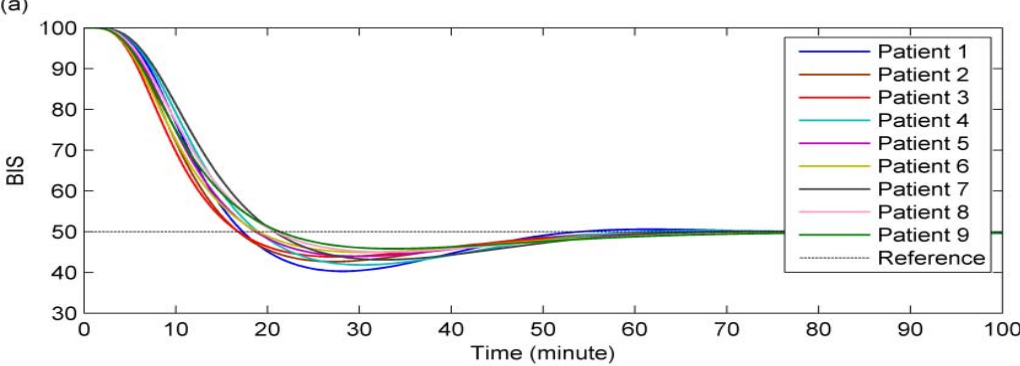

(b)

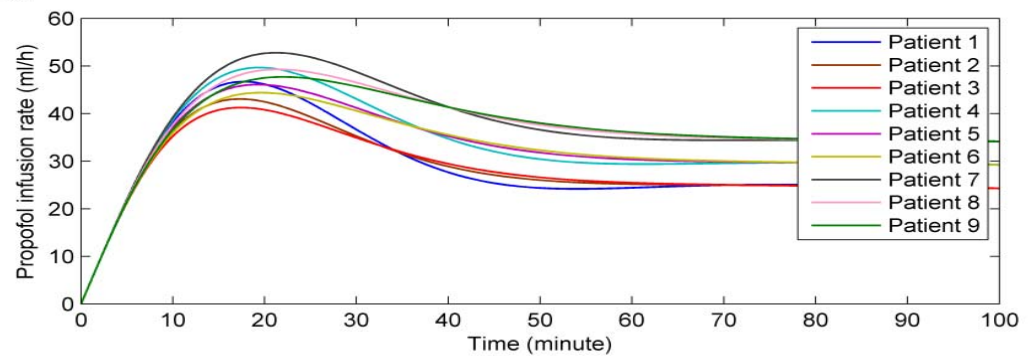

Figure 2. Performance of the controller without initial bolus. (a) BIS response (b) Propofol infusion rate

The induction phase can be greatly reduced when an initial bolus of propofol was administered. Due to the presence of closed-loop control, a lower-than-clinical practice bolus injection was administered during the initial phase, i.e., $250 \mathrm{ml} / \mathrm{h}$ at the first minute. Then, the controller was initiated in the second minutes. 
Table 3 and Figure 3 presents the simulation result of the controller with an initial bolus for all patients. The result shows that all BIS converges to the targeted value. The mean ID was reduced to $2.36 \mathrm{~min}$. All BIS reading reached 60 within $3.03 \mathrm{~min}$. However, the mean OS was shown to increase from $13.01 \%$ to $16.60 \%$

Table 3. Performance of the controller with initial bolus

\begin{tabular}{cccc}
\hline Patient no. & ID $(\mathrm{min})$ & OS $(\%)$ & IAE \\
\hline 1 & 2.83 & 19.22 & 14047 \\
2 & 2.08 & 20.07 & 12855 \\
3 & 1.83 & 27.79 & 12291 \\
4 & 3.00 & 14.54 & 1843 \\
5 & .17 & 12.46 & 10520 \\
6 & 1.83 & 21.28 & 9944 \\
7 & 3.33 & 11.55 & 10477 \\
8 & 2.25 & 7.36 & 7975 \\
9 & 1.92 & 15.15 & 7871 \\
Mean & 2.36 & 16.60 & 10869 \\
\hline
\end{tabular}
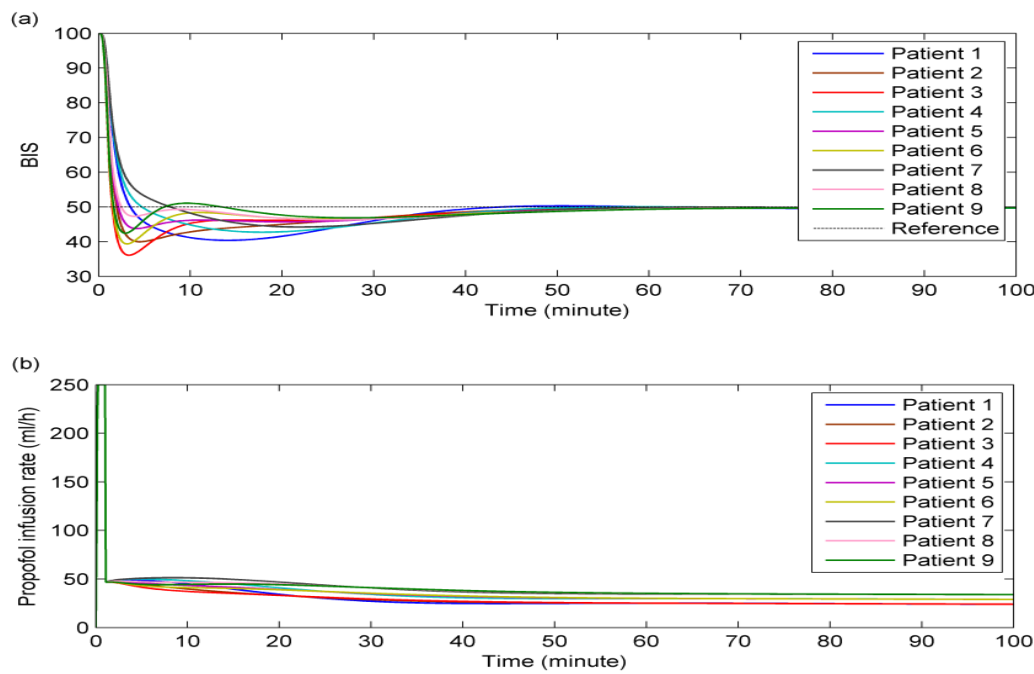

Figure 3. Performance of the controller with initial bolus. (a) BIS response (b) Propofol infusion rate

While the ID can also be shortened by increasing the $k_{i}$ value, it will also cause a large overshoot which may put the patient safety at risk. Besides these two conflicting performance criteria (ID and OS), the performance of the controller has to be compromised for system robustness if the BIS response were to be kept within the range of 40-60 for all patients. This shows that the inter-individual variability is a great challenge in the design of a safe and efficient controller for anaesthesia system.

\section{CONCLUSION}

An observer-based state feedback control with integral action was designed to regulate the BIS signal using propofol infusion rate. The state feedback gain and observer gain were designed using the LP approach and took into consideration the positiveness of states as well as the uncertainty of the PK model. Simulation result shows that all BIS was able to converge to the set-point, but with a long induction phase. A bolus injection of propofol during the initial stage was able to reduce the induction phase, but will increase the overshoot percentage. This shows that the design of a fixed controller for the whole population is difficult due to the large inter-individual variability exists among patients. Individualised controller may be the key to tackle patient variability in order to realise the closed-loop control of anaesthesia.

\section{REFERENCES}

[1] H. Derendorf and B. Meibohm, "Modeling of Pharmacokinetic/Pharmacodynamic (PK/PD) Relationships: Concepts and Perspectives," Pharmaceutical Research, vol. 16(2), pp. 176-85, Feb 1999. 
[2] L. Farina and S. Rinaldi, "Positive Linear Systems: Theory and Applications," Canada: John Wiley \& Sons, 2000.

[3] S. Yelneedi, "Advanced Control Strategies for Automatic Drug Delivery to Regulate Anaesthesia during Surgery," PhD thesis, National University of Singapore, 2009.

[4] T. Kaczorek, "Stabilization of Positive Linear Systems by State-Feedbacks," Pomiary, Automatyka, Kontrola pp. 2$5,1999$.

[5] P.D. Leenheer, and D. Aeyels, "Stabilization of Positive Linear Systems," Systems \& Control Letter, vol. 44 (4), pp. 259-271, 2001

[6] S.P. Boyd, et al, "Linear Matrix Inequalities in System and Control Theory," Philadelphia: SIAM, 1994.

[7] H. Gao, J. Lam, C. Wang, and S. Xu, "Control for Stability and Positivity: Equivalent Conditions and Computation," IEEE Transactions on Circuits and Systems II: Express Briefs, vol. 52 (9), pp. 540-544, 2005.

[8] M. Rami and F. Tadeo, "Controller Synthesis for Positive Linear Systems With Bounded Controls," IEEE Transactions on Circuits and Systems II: Express Briefs, vol. 54 (2), pp. 151-155, 2007.

[9] M. Rami, "Solvability of Static Output-Feedback Stabilization for LTI Positive Systems," Systems \& Control Letters, vol. 60 (9), pp. 704-708, 2011.

[10] M. Bolajraf, M. Rami and U.R. Helmke, "Robust Positive Interval Observers for Uncertain Positive Systems," 18th IFAC World Congress, vol. 44 (1), pp. 14330-4, 2011.

[11] H.M. Soliman and M. Soliman, "Design of Observer-based Robust Power System Stabilizers", International Journal of Electrical and Computer Engineering, vol. 6 (5), pp. 1956-1966, 2016.

[12] P. Ramana, K.A. Mary and M.S. Kalavathi, "State Feedback Linearization of a Non-linear Permanent Magnet Synchronous Motor Drive”, Indonesian Journal of Electrical Engineering and Computer Science, vol.1 (3), pp. 534$542,2016$.

[13] K. Chakraborty, S.S. Ghosh, R.D. Basak and I. Roy, "Temperature Control of Liquid Filled Tank System using Advance State Feedback Controller", TELKOMNIKA Indonesian Journal of Electrical Engineering, vol. 14 (2), pp. 288-292, 2015.

[14] T. Schnider, et al., "The Influence of Age on Propofol Pharmacodynamics," Anesthesiology, vol. 90 (6), pp. 1502$1516,1999$.

[15] T. Schnider, et al., "The Influence of Method of Administration and Covariates on the Pharmacokinetics of Propofol in Adult Volunteers," Anesthesiology, vol. 88 (5), pp. 1170-1182,1998.

[16] J. Gouzé, A. Rapaport, and M. Hadj-Sadok, "Interval Observers for Uncertain Biological Systems,” Ecological Modelling, vol. 133 (12), pp. 45-56, 2000.

[17] D. Luenberger, “An Introduction to Observers," IEEE Transactions on Automatic Control, vol. 16 (6), pp. 596-602, 1971.

[18] K. Soltesz, G. Dumont, and J. Ansermino, "Assessing Control Performance in Closed-loop Anesthesia," In 21st Mediterranean Conference on Control Automation (MED), pp. 191-196, 2013.

[19] N. Liu, et al., "Titration of Propofol for Anesthetic Induction and Maintenance Guided by the Bispectral Index: Closed-loop versus Manual Control: A Prospective, Randomized, Multicenter Study,” Anesthesiology, vol. 104, pp. 686-695, 2006.

[20] S. Tarbouriech, I. Queinnec, G. Garcia, and M. Mazerolles, "Control of Anesthesia Based on Singularly Perturbed Model." In F. Cacace, L. Farina, R. Setola, A. Germani, editors. Positive Systems. POSTA 2016. Lecture Notes in Control and Information Sciences, vol 471, 2017, Springer.

\section{BIOGRAPHIES OF AUTHORS}

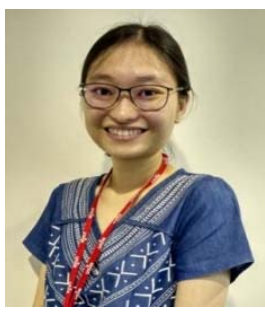

Chang Jing Jing is with Department of Computer and Communication Technology, University Tunku Abdul Rahman, Malaysia. She received her Ph.D. in Control and Automation Engineering in Universiti Putra Malaysia. Her research interests include modelling, control, artificial intelligence and biomedical engineering.

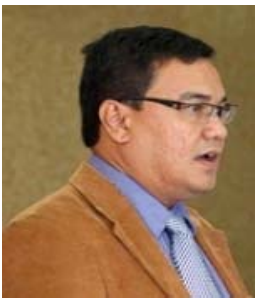

S. Syafiie received his DEA and PhD degrees from University of Valladolid, Spain in 2004 and 2007, respectively, in the area of systems engineering and automatic control. In 2007, he continue as postdoctoral researcher in the area of continuous action reinforcement learning in the same university. In 2008, he did his post doctoral research in Gent University, Belgium in the area of biomedical engineering and machine learning. From 2009 to 2017 he was appointed as senior lecturer at department chemical and environmental engineering Universiti Putra Malaysia. In 2017 he joined industrial engineering department in Syiah Kuala University. His research interests include machine learning and control theory application in biomedical engineering, chemical engineering and supply chain management. 